\title{
3D QSAR Selectivity Analyses of Carbonic Anhydrase Inhibitors: Insights for the Design of Isozyme Selective Inhibitors
}

\section{(Supporting Information)}

Alexander Weber ${ }^{1,4}$, Markus Böhm ${ }^{3}$, Claudiu T. Supuran ${ }^{2} *$, Andrea Scozzafava ${ }^{2}$, Christoph A. Sotriffer ${ }^{1}$, and Gerhard Klebe ${ }^{1 *}$

${ }^{1}$ Department of Pharmaceutical Chemistry, University of Marburg, Marbacher Weg 6, D35032 Marburg, Germany.

${ }^{2}$ Università degli Studi di Firenze, Dipartimento di Chimica, Laboratorio di Chimica Bioinorganica, Via della Lastruccia, 3, Rm. 188, I-50019 Sesto Fiorentino (Florence), Italy.

${ }^{3}$ Pfizer Global R\&D, Eastern Point Road, Groton, Connecticut 06340

${ }^{4}$ Discovery IT, ALTANA Pharma AG, Konstanz, Germany.

* Corresponding Authors, Phone: +49 642128 21313, Fax: +49 642128 28994, E-mail: klebe@mailer.uni-marburg.de (GK) and Phone: +39-055-4573005, Fax: +39-055-4573385, E-mail: claudiu.supuran@unifi.it (CTS). 
Tab. S01) Parameters of the grid boxes used for CoMFA and CoMSIA analyses.

\begin{tabular}{|c|c|c|c|c|c|c|}
\hline & \multicolumn{3}{|c|}{$1 \AA$ Grid } & \multicolumn{3}{|c|}{$2 \AA$ Grid } \\
\hline & $\mathrm{x}$ & $\mathrm{y}$ & $\mathrm{Z}$ & $\mathrm{x}$ & $\mathrm{y}$ & $\mathrm{Z}$ \\
\hline Lower corner & -15.8 & -23.5 & -11.0 & -15.8 & -23.5 & -11.0 \\
\hline Higher corner & 10.0 & 10.2 & 13.4 & 10.0 & 10.2 & 13.4 \\
\hline Grid Spacing & 1.0 & 1.0 & 1.0 & 2.0 & 2.0 & 2.0 \\
\hline Numer of steps & 26 & 34 & 25 & 13 & 17 & 13 \\
\hline Grid points & \multicolumn{3}{|c|}{22100} & \multicolumn{3}{|c|}{2873} \\
\hline
\end{tabular}


Tab. S02) Distances between atoms that are involved in a hydrogen bond network in CA I and CA II. For each isozyme, one complex structure (CA I: 1azm, 2.0 Å resolution, A_18; CA II: 1cil, $1.6 \AA$, B_05) and one apo structure (CA I: 2cab, $2.0 \AA$; CA II: 2 cba, $1.54 \AA$ ) were selected to determine the distances.

\begin{tabular}{cccc}
\hline CA I & Atomtyp & Distance (2cab) & Distance (1azm) \\
\hline His94 - Gln92 & ND1 - OE1 & $2.7 \AA$ & $2.8 \AA$ \\
Gln92 - Asn69 & NE1 - OD1 & $3.1 \AA$ & $3.2 \AA$ \\
Asn69 - Glu58 & ND2 - OE1 & $3.5 \AA$ & $3.6 \AA$ \\
\hline CA II & Atomtyp & Distance (2cba) & Distance (1cil) \\
\hline His94 - Gln92 & ND1 - OE1 & $2.7 \AA$ & $2.8 \AA$ \\
Glu69 - Arg58 & OE2 - NH2 & $3.1 \AA$ & $3.0 \AA$ \\
Glu69 - Arg58 & OE1 - NE & $2.7 \AA$ & $2.7 \AA$ \\
Glu69 - Arg58 & OE1 - NH2 & $3.1 \AA$ & $3.2 \AA$ \\
\hline
\end{tabular}

